\title{
GMR
}

\section{Expression of GRIN2A in benign and malignant nasopharyngeal diseases and its clinical significance}

\author{
X.J. Zhou' ${ }^{1}$ B.R. Lin ${ }^{1}$ and Y. Wang ${ }^{2}$ \\ ${ }^{1}$ Otolaryngology Department, \\ Zhongshan Hospital Affiliated to Guangzhou University of Chinese Medicine, \\ Zhongshan, China \\ ${ }^{2}$ Pathology Department, \\ Zhongshan Hospital Affiliated to Guangzhou University of Chinese Medicine, \\ Zhongshan, China \\ Corresponding author: X.J. Zhou \\ E-mail: zd1232@126.com \\ Genet. Mol. Res. 14 (4): 17289-17295 (2015) \\ Received August 18, 2015 \\ Accepted October 18, 2015 \\ Published December 16, 2015 \\ DOI http://dx.doi.org/10.4238/2015.December.16.29
}

ABSTRACT. The gene glutamate receptor, ionotropic, N-methyl D-aspartate 2A (GRIN2A) is associated with development and neuron viability, and our previous studies showed it to be substantially methylated in nasopharyngeal carcinoma, indicating a link to this disease. The aim of this work was to investigate GRIN2A expression and its clinical significance in nasopharyngeal carcinoma, in contrast to nasopharyngitis and nasopharyngeal precancerous lesions. Fifty patients with nasopharyngeal carcinoma were selected as study subjects, while 28 chronic nasopharyngitis patients and 22 individuals with nasopharyngeal precancerous lesions were used as controls. Immunohistochemical analysis was used to study GRIN2A protein expression, and its relationship with nasopharyngeal carcinoma clinical stage and histopathological features were assessed. GRIN2A appeared as yellow staining in the cytoplasm or nucleus. It was strongly expressed in the nasopharyngeal epithelial tissues of patients with 
chronic nasopharyngitis and in nasopharyngeal precancerous lesions, the proportions of GRIN2A-positive cells being 82.1 and $72.7 \%$, respectively. However, it was weakly expressed in nasopharyngeal carcinoma tissues, with $28.0 \%$ of cells testing positive $(P<0.001)$. No significant difference in the expression of GRIN2A was observed between different clinical stages and pathological grades. We conclude that weak GRIN2A expression is a major feature of nasopharyngeal carcinoma.

Key words: Nasopharyngeal carcinoma; GRIN2A

\section{INTRODUCTION}

The N-methyl-D-aspartate glutamate receptor subunit GRIN2A is involved in development, neuron viability, study, and memory, but is rarely associated with cancer. Our previous studies (Zhou et al., 2013) have shown that the glutamate receptor, ionotropic, N-methyl D-aspartate 2A (GRIN2A) gene is substantially methylated in both nasopharyngeal carcinoma and colon cancer (Kim et al., 2008). Further investigation is necessary to verify the correlation between GRIN2A and nasopharyngeal carcinoma, and the clinical stages and pathological grades of this disease. In the current study therefore, we used immunohistochemistry to analyze GRIN2A protein expression in nasopharyngeal carcinoma samples, using tissues affected by nasopharyngitis and nasopharyngeal precancerous lesions as controls.

\section{MATERIAL AND METHODS}

\section{Patients and tissue samples}

Nasopharyngeal carcinoma, nasopharyngitis, and nasopharyngeal precancerous lesion samples were selected from a specimen database using a random number table. All tissues came from individuals having been outpatients of the Zhongshan Hospital affiliated to Guangzhou University of Chinese Medicine between 2010 and 2014. Fifty cases of nasopharyngeal carcinoma were included, comprising 31 men and 19 women with an average age of $36.6 \pm 12.9$ years (mean \pm standard deviation). Histological types were as follows: 38 cases of nonkeratinizing carcinoma, eight undifferentiated cancers, and four mixed nonkeratinizing/undifferentiated cancers. The following clinical stages of nasopharyngeal carcinoma were observed: 19 cases of stage I, 20 of stage II, nine of stage III, and two of stage IV. There were 28 cases of nasopharyngitis, including 16 men and 12 women with an average age of $35.3 \pm 10.7$ years. In addition, 22 cases of nasopharyngeal precancerous lesions were included, consisting of 12 men and 10 women with an average age of $36.3 \pm 11.7$ years.

Nasopharyngeal carcinoma was diagnosed based on paraffin-embedded tissue slices, as per convention. Cancer stage was determined using the clinical staging criteria proposed in 2008 (Committee of Chinese Clinical Staging of Nasopharyngeal Carcinoma, 2009), and involved conventional endoscopy of the nasopharynx, systemic ultrasonography, and nasopharyngeal computed tomography or magnetic resonance imaging to analyze proliferation and metastasis. Pathological categorization of nasopharyngeal carcinoma was based on the upper respiratory tract and ear histological classification criteria (Shanmugaratnam and Sobin, 1993) proposed by the 
World Health Organization in 1991. Following the standards proposed by the Cancer Institute of Guangdong Zhongshan University (Zong et al., 2001), nasopharyngeal precancerous lesions were defined as atypical squamous metaplasia or dysplasia in the nasopharyngeal epithelium. We used the following criteria to identify atypical tissues and cells: a significantly increased basal cell layer, varied cell sizes, poor differentiation, an aberrantly large nucleus, a prominent nucleolus, common karyokinesis, an increased ratio of nucleus to cytoplasm, and disturbed or absent cell polarity.

\section{Reagents and equipment}

A rabbit anti-human GRIN2A monoclonal antibody (catalog No. WA-0322R) was purchased from Beijing Pherobio Technology Co., Ltd. (Beijing, China), while a MaxVision Immunohistochemistry Kit (mouse/rabbit, catalog No. KIT-5010/5020/5030) was purchased from Fuzhou Maixin Biotechnology Co., Ltd. (Fuzhou, China). Antigen heat-repairing reagents (catalog No. C-0017) were purchased from Beijing Bioss Biosynthesis Biotechnology Co., Ltd. (Beijing, China).

\section{Methods}

Samples incubated with phosphate-buffered saline in place of the primary antibody were used as negative controls, and a biopsy specimen known to contain nasopharyngeal carcinoma tissue served as a positive control. The acidic repair method was used for antigen retrieval. Firstly, samples were incubated with $3 \%$ concentrate hydrogen peroxide and $1 \%$ concentrate citrate buffer, $\mathrm{pH} 6.0$, in a sealed container inside a water bath at $92^{\circ}-95^{\circ} \mathrm{C}$ for $15 \mathrm{~min}$, then allowed to cool down to room temperature. Subsequently, they were incubated with goat serum at $37^{\circ} \mathrm{C}$ for 20 min in a sealed container before being exposed to the primary antibody at $4^{\circ} \mathrm{C}$ overnight. The staining protocol was strictly in accordance with the instructions provided by the immunohistochemistry kit manufacturer.

\section{Immunohistochemical criteria and statistical methods}

Cells displaying light-yellow-to-brown particles in the cytoplasm or nucleus were defined as positive cells. Under an optical microscope at 400× magnification, five visual fields were randomly selected for each slice and the numbers of positive and negative cells were recorded. The positive rate was then calculated as follows (Wang et al., 2013): number of positive cells $\times 100 \% \div$ (number of negative cells + number of positive cells). Simultaneously, the intensity of staining was scored for each slice: 0 was assigned for no staining, 1 for light yellow, 2 for deep yellow, and 3 for brown. We calculated the positive expression value as the positive rate multiplied by the staining intensity score. The grading criteria were as follows: when positive expression was $<0.5$ with extremely week protein expression that could be considered as absent of protein expression, and that was recorded as grade 1 ; a value $\geq 0.5$ but $<1$ with weak protein expression was considered grade 2 ; a value $\geq 1$ and $<1.5$ with moderate protein expression was noted as grade 3 ; and a value $\geq 1.5$ with strong protein expression was classed as grade 4.

SPSS 19.0 software (IBM Corp., Armonk, NY, USA) was used for data processing. A nonparametric test (the Kruskal-Wallis test of $k$ independent samples) was used for statistical analysis and pairwise comparisons were made using the Bonferroni method. Differences with $\mathrm{P}$ values $<0.05$ were considered statistically significant. 


\section{RESULTS}

\section{GRIN2A expression in nasopharyngeal carcinoma, nasopharyngeal precancerous lesions, and nasopharyngitis}

GRIN2A protein expression was mainly concentrated in the cytoplasm but was also detected in the nucleus. Depending on the expression level, this protein appeared in our immunohistochemical assay as pale yellow, dark yellow, or brown (Figure 1).
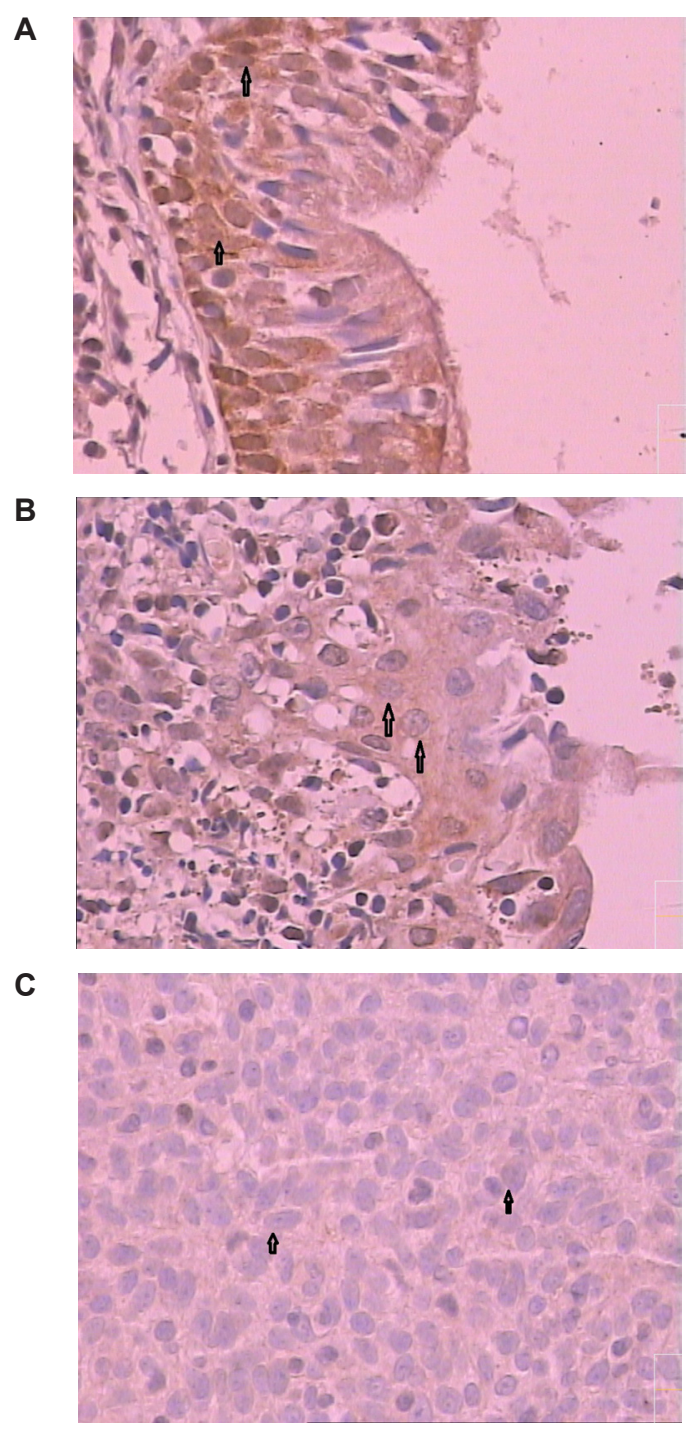

Figure 1. (A) Nasopharyngitis, (B) nasopharyngeal precancerous lesion, and (C) nasopharyngeal carcinoma samples (400X magnification) showing strong (grade 4), moderate (grade 3), and absent (grade 1) GRIN2A expression, respectively. 
GRIN2A was strongly expressed in the epithelial tissues of patients with chronic nasopharyngitis and moderate expressed in nasopharyngeal precancerous lesions, being detected in many cells and at a high concentration. This was evident from the brown staining and positive rates of 82.1 and $72.7 \%$, respectively. In contrast, this protein was only weakly expressed in nasopharyngeal carcinoma tissue, with a positive rate of $28.0 \%$ (Table 1). Pairwise comparisons revealed significant differences in GRIN2A expression between nasopharyngeal carcinoma and both nasopharyngitis and nasopharyngeal precancerous lesions, but no significant variation was observed between these two latter conditions.

Table 1. GRIN2A expression in nasopharyngeal carcinoma, nasopharyngeal precancerous lesions, and nasopharyngitis.

\begin{tabular}{lrrrrr}
\hline & \multicolumn{4}{c}{ GRIN2A positive expression (cases) } \\
\cline { 2 - 6 } & $\mathrm{N}$ & Grade 1 & Grade 2 & Grade 3 & Grade 4 \\
\hline Nasopharyngeal carcinoma $^{\mathrm{a}}$ & 50 & 36 & 10 & 3 & 1 \\
Nasopharyngeal precancerous lesions $^{\mathrm{b}}$ & 22 & 6 & 2 & 2 & 12 \\
Chronic nasopharyngitis $^{\mathrm{c}}$ & 28 & 5 & 2 & 11 & 10 \\
\hline
\end{tabular}

Chi-square $=36.307 ; P=0.000$. Chi-square $(a$ and $b)=17.78 ; P(a$ and $b)=0.000$. Chi-square $(a$ and $c)=32.43 ; P(a$ and $c)=0.000$. Chi-square $(b$ and $c)=0.584 ; P(b$ and $c)=0.445$.

\section{GRIN2A protein expression at different clinical stages and in different pathological types of nasopharyngeal carcinoma}

These data are shown in Tables 2 and 3. No significant differences in GRIN2A expression were observed between different clinical stages or types of nasopharyngeal carcinoma.

Table 2. GRIN2A protein expression at different clinical stages of nasopharyngeal carcinoma.

\begin{tabular}{lrrrrr}
\hline & \multicolumn{4}{c}{ GRIN2A positive expression (cases) } \\
\cline { 2 - 6 } & $\mathrm{N}$ & Grade 1 & Grade 2 & Grade 3 & Grade 4 \\
\hline Stage I & 19 & 16 & 2 & 3 & 1 \\
Stage II & 20 & 15 & 2 & 0 & 0 \\
Stage III & 9 & 5 & 2 & 0 & 0 \\
Stage IV & 2 & 0 & 2 & \\
\hline
\end{tabular}

Chi-square $=5.849 ; P=0.119$.

Table 3. GRIN2A protein expression in different pathological types of nasopharyngeal carcinoma.

\begin{tabular}{lrrccc}
\hline & \multicolumn{4}{c}{ GRIN2A positive expression (cases) } \\
\cline { 2 - 6 } & $\mathrm{N}$ & Grade 1 & Grade 2 & Grade 3 & Grade 4 \\
\hline Nonkeratinizing carcinoma & 38 & 28 & 6 & 3 & 1 \\
Mixed & 4 & 3 & 1 & 0 & 0 \\
Undifferentiated cancer & 8 & 5 & 3 & 0 & 0 \\
\hline
\end{tabular}

Mixed $=$ mixed nonkeratinizing/undifferentiated cancer. Chi-square $=0.221 ; \mathrm{P}=0.895$.

\section{DISCUSSION}

GRIN2A, encoding the N-methyl-D-aspartate (NMDA) receptor 2A subunit, and also known as NMDAR2A or GR2A, is located in chromosomal region 10q11. Its GenBank accession 
No. is NM_000833, and the full-length gene and mRNA transcript consist of $421.191 \mathrm{~kb}$ and 6293 bp, respectively.

NMDA receptors (NRs) are ionotropic excitatory amino acid receptors coupled with a $\mathrm{Ca}^{2+}$ channel, and consist of seven subtypes (NRI, NR2A, NR2B, NR2C, NR2D, NR3A, and NR3B). It is believed that these receptors are predominantly distributed across the central nervous system and play an important role in development, neuronal activity adjustment, dendrite and axon growth, and synaptic plasticity. They also perform an essential function in the formation of neuronal circuits, and in learning and memory. Their reduced functionality can cause developmental delay, while elevated activity may lead to neuronal damage. Further studies have indicated that NRs, such as GRIN2A, are not only related to nervous system lesions, but also malignant tumors (including those affecting other parts of the body). In one study, GRIN2A was found to be mutated in $33 \%$ of malignant melanomas (Wei et al., 2011), and its mutation has been shown to shorten the survival time of patients with such cancers (D'mello et al., 2014). It is also substantially methylated in colon cancer tissue and may represent a tumor suppressor gene (Kim et al., 2008).

The results of our study show that the GRIN2A protein is rarely produced in nasopharyngeal carcinoma, while being expressed at elevated levels in tissues affected by chronic nasopharyngitis and nasopharyngeal precancerous lesions. Moreover, this difference was statistically significant. Pairwise comparisons showed no significant variation in GRIN2A expression between chronic nasopharyngitis and nasopharyngeal precancerous lesions, but did reveal statistically significant differences between each of these and nasopharyngeal carcinoma, indicating that loss of GRIN2A expression occurs only in the latter condition. In addition, our results indicated no significant differences in the expression of this protein between pathological types or clinical stages of nasopharyngeal carcinoma. These findings suggest that weak GRIN2A expression is one of the characteristics of nasopharyngeal cancer, and does not significantly change as this disease progresses.

Nasopharyngeal carcinomas are more pervasive in southern China than in other regions of the world. Compared to other cancers, these carcinomas are more strongly associated with environmental factors than with other pathogenic influences such as heredity or Epstein-Barr virus. It is of note that this malignancy is the only such named after a geographical region, being also known as Canton tumor. Previous studies of nasopharyngeal carcinoma have emphasized genetic elements such as oncogenes, but tumorigenesis and tumor progression are usually driven by an interaction between genetic and epigenetic changes. Carcinogenesis is a pathological process involving multiple genes and multistage mutation accumulation, in which inactivation of tumor suppressor genes is a key factor. There exist many mechanisms by which such genes lose their functionality. Of these, DNA methylation is the third most common process, after gene deletion and mutation, leading to inactivation of tumor suppressor genes. In some cases, DNA methylation is the sole cause of such gene silencing (Herman and Baylin, 2003), making it a hot spot in nasopharyngeal carcinoma studies. The genes RRAD, PCDH8, and WWOX are considered tumor suppressors and have been found to be weakly expressed in nasopharyngeal carcinoma tissue following their methylation (He et al., 2012; Mo et al., 2012; Yang et al., 2014), indicating that this epigenetic process is involved in such malignancies. Our previous study (Zhou et al., 2013) also showed substantial methylation of GRIN2A after the development of nasopharyngeal carcinoma, and suggested that promoter methylation weakens GRIN2A expression in this disease. In conclusion, this mechanism is a major feature of nasopharyngeal carcinoma, and plays an important role in its development. 


\section{Conflicts of interest}

The authors declare no conflict of interest.

\section{ACKNOWLEDGMENTS}

\#81072847).

\section{REFERENCES}

Committee of Chinese Clinical Staging of Nasopharyngeal Carcinoma (2009). Report on the revision of nasopharyngeal carcinoma '92 staging. Chin. J. Radiat. Oncol. 18: 2-6.

D'mello SA, Flanagan JU, Green TN, Leung EY, et al. (2014). Evidence that GRIN2A mutations in melanoma correlate with decreased survival. Front. Oncol. 3: 333.

He D, Zeng Q, Ren G, Xiang T, et al. (2012). Protocadherin8 is a functional tumor suppressor frequently inactivated by promoter methylation in nasopharyngeal carcinoma. Eur. J. Cancer Prev. 21: 569-575.

Herman JG and Baylin SB (2003). Gene silencing in cancer in association with promoter hypermethylation. N. Engl. J. Med. 349: 2042-2054.

Kim MS, Chang X, Nagpal JK, Yamashita K, et al. (2008). The N-methyl-D-aspartate receptor type 2A is frequently methylated in human colorectal carcinoma and suppresses cell growth. Oncogene 27: 2045-2054.

Mo Y, Midorikawa K, Zhang Z, Zhou X, et al. (2012). Promoter hypermethylation of Ras-related GTPase gene RRAD inactivates a tumor suppressor function in nasopharyngeal carcinoma. Cancer Lett. 323: 147-154.

Shanmugaratnam K and Sobin LH (1993). World Health Organization histological classification of tumours of the upper respiratory tract and ear. A commentary on the second edition. Cancer 71: 2689-2697.

Wang Y, Zhou XJ,Wang SQ, Xuan RX, et al. (2015). Study on the correlation of disintegrin metalloproteinase 23 expression on nasopharyngeal carcinoma with its clinical stages. J. Guangxi Med. Univ. 32: 44-46.

Wei X, Walia V, Lin JC, Teer JK, et al. (2011). Exome sequencing identifies GRIN2A as frequently mutated in melanoma. Nat. Genet. 43: 442-446.

Yang Z, Lan H, Chen X, Li P, et al. (2014). Molecular alterations of the WWOX gene in nasopharyngeal carcinoma. Neoplasma 61: 170-176.

Zhou XJ, Chen JH, Wang SZ, Wang SQ, et al. (2014). Study on DNA methylation features of ADAM23, MIMT1, FAM150B, GRIN2A and LRRC4 before and after nasopharyngeal carcinoma onset. J. Chongqing Med. Univ. 39: 341-344.

Zong YS, Wu QL, Liang XM, Liang YJ, et al. (2001). The study of histological type and precancerous lesions of nasopharyngeal carcinoma. Ai Zhen 20: 117-127. 